\title{
Eficacia de las intervenciones basadas en cuidados paliativos en pacientes y cuidadores
}

\author{
Efficacy of palliative care interventions in patients and caregivers
}

\section{Objetivos}

Determinar la eficacia de la implementación de intervenciones de cuidados paliativos sobre: 1) la sobrevida, la calidad de vida y la sintomatología de pacientes con enfermedades terminales; 2) la calidad de vida de sus cuidadores.

\section{Métodos}

Se realizó una búsqueda en MEDLINE, EMBASE, CINAHL y Cochrane CENTRAL de estudios publicados hasta Julio 2016. Los artículos fueron evaluados de manera independiente por dos investigadores. Los desacuerdos fueron resueltos por consenso con otros dos investigadores. Criterios de inclusión: ensayos clínicos controlados y aleatorizados que hubieran reclutado pacientes mayores de 18 años con enfermedad terminal y hubieran reportado al menos un resultado (calidad de vida, sintomatología, estado de ánimo, sobrevida, planificación de cuidados, lugar de muerte, utilización de recursos, costos en salud, satisfacción con el cuidado). Estos pacientes debían haber recibido una intervención paliativa que hubiera abarcado más de dos dominios. El riesgo de sesgo fue evaluado por dos investigadores utilizando la herramienta de Cochrane en forma separada para los resultados objetivos y los subjetivos. Todos los estudios fueron incluidos en
Kavalieratos D y col. JAMA, 2016;316(20):2104-14 la síntesis narrativa. Los resultados de calidad de vida, sintomatología y sobrevida fueron combinados mediante meta-análisis. La sobrevida fue expresada en todos los estudios mediante Hazard Ratios (HR). Para evaluar la heterogeneidad entre los diferentes estudios se utilizó el I2, el T2 y la Q de Cochrane.

\section{Resultados}

Fueron identificados 6.158 registros únicos, de los cuales 200 fueron potencialmente relevantes para ser analizados. 56 artículos fueron incluidos en la síntesis narrativa (43 ensayos con un total de 12.731 pacientes con una edad media de 67 años y 2.479 cuidadores). La enfermedad más prevalente fue cáncer (69,7\%), y el $41,8 \%$ de los estudios tomaron lugar en el domicilio. Los cuidados paliativos se asociaron a mejoras en la planificación de los cuidados, mayor satisfacción con el cuidado y menor utilización de recursos. Como lo muestra la tabla 1, cuando a pesar de su heterogeneidad, se meta-analizaron todos los estudios, se observaron diferencias estadísticamente significativas y clínicamente relevantes en la calidad de vida entre el primero y el tercer mes de la intervención. Sin embargo, estos hallazgos fueron más débiles cuando se incluyeron en el meta-análisis solo los estudios con bajo riesgo de sesgo.

Tabla 1. Eficacia de las intervenciones basadas en cuidados paliativos sobre la calidad de vida y la sintomatología de los pacientes.

\begin{tabular}{l|c|c|c|c|c|}
\multicolumn{1}{c|}{ Dimensión } & Escala & $\begin{array}{c}\text { Diferencia } \\
\text { clinicamente } \\
\text { relevante }\end{array}$ & \multicolumn{2}{|c|}{ Diferencia entre el primero y el tercer mes de la intervención } \\
\cline { 4 - 5 } & & 9 puntos & Total de los estudios & Solo los estudios con bajo riesgo de sesgo \\
Calidad de vida & FACIT-Pal & $+11,36$ puntos & $+4,94$ puntos \\
& $(0$ a 184$)$ & 5,7 puntos & DME $0,46(0,08$ a 0,83$)$ & DME $0,20(0,06$ a 0,34$)$ \\
\hline Sintomatología & ESAS & & $-10,3$ puntos & $-3,28$ puntos \\
& $(0$ a 90$)$ & & DME $-0,66(-1,25$ a $-0,07)$ & DME $-0,21(-0,42$ a 0,0$)$ \\
\hline
\end{tabular}

Luego de los tres meses, no se observaron diferencias clínicamente relevantes en la calidad de vida ni en la sintomatología, ni tampoco en la sobrevida entre ambos grupos (HR 0,90; IC95\% 0,69 a 1,17; $1275,3 \%)$.

\section{Conclusiones}

Los cuidados paliativos mostraron cierta efectividad para mejorar la calidad de vida y la carga sintomática de pacientes con enfermedades terminales a corto plazo. Sin embargo esta evidencia es más débil cuando proviene solo de los estudios con bajo riesgo de sesgo.

\section{Comentario}

Los cuidados paliativos intentan acompañar a la persona que transita estadios avanzados de una enfermedad terminal o no curable y a su entorno familiar, buscando optimizar la forma de vivir satisfaciendo las necesidades físicas, psicoemocionales y espirituales. No sólo están destinados a la etapa final de la vida, si no que pueden acompañar también al paciente en el desarrollo fluctuante de una enfermedad, asistiendo durante el curso de tratamientos activos y suspendiendo el seguimiento durante períodos libres de enfermedad ${ }^{1}$. La práctica de los médicos de atención primaria se caracteriza por el seguimiento longitudinal de sus pacientes, con un rol en el acompañamiento y tratamiento de enfermedades crónicas en etapas tempranas que eventualmente se convertirán en terminales. Una adecuada planificación de los cuidados que se llevarán a cabo en estadios avanzados de la enfermedad permite equilibrar los objetivos de tratamiento y los valores del paciente con la comprensión del equipo de salud de la situación clínica y el pronóstico. Además, la modalidad de trabajo de los médicos de familia puede tener un fuerte impacto en la calidad de los cuidados de fin de vida, ya que su rol puede facilitar que el sistema de salud se centre en un enfoque integrado en la atención preventiva, curativa y paliativa, y no solamente en la respuesta a las exacerbaciones agudas de la enfermedad en la central de emergencias. ${ }^{3}$ Este meta-análisis se llevó a cabo para evaluar la eficacia de los cuidados paliativos sobre diferentes medidas de resultado, concluyendo que estos dispositivos contribuyen a mejoras en la calidad de vida y la carga sintomática de pacientes con enfermedades terminales. Sin embargo, existió gran heterogeneidad entre los estudios, y se combinaron individuos con diferentes enfermedades, distintos estadios, distintos ámbitos de trabajo y una gran variabilidad de intervenciones, cuyos resultados fueron medidos con herramientas diversas. En cierta manera, es debatible cuán combinables eran los estudios incluidos en el metaanálisis y, por lo tanto, la validez y extrapolabilidad de sus resultados a la población general.

\section{Conclusiones del comentador}

Si bien todavía existen limitaciones respecto de la evidencia disponible, para los pacientes en situación de fin de vida o con enfermedades terminales, las intervenciones basadas en cuidados paliativos parecen contar con una evidencia moderada que las avala.

María Celia Iñurrategui [ Servicio de Medicina Familiar y Comunitaria. Hospital Italiano de Buenos Aires. maria.inurrategui@ hospitalitaliano.org.ar] Iñurrategui MC. Eficacia de las intervenciones basadas en cuidados paliativos en pacientes y cuidadores. Evid Act Pract Ambul 2018;21(2):52. Comentado de: Kavalieratos D y col. Association Between Palliative Care and Patient and Caregiver Outcomes: A Systematic Review and Meta-analysis. JAMA, 2016;316(20):2104-14.

\section{Referencias}

1. Servicio de Medicina Familiar y Comunitaria. "Medicina Familiar y práctica ambulatoria". $3^{\text {a }}$ Edición. Editorial Panamericana, Buenos Aires 2016. 2. Ghosh A., y col. Interaction of Palliative Care and Primary Care. Clinics in Geriatric Medicine 2015, 31(2), 207-218. 